\title{
Metagenomics Analysis of Thrombus Samples Retrieved from Mechanical Thrombectomy
}

\author{
Atulabh Vajpeyee, MD,DM, FINS ${ }^{1}$, Puneet Singh Chauhan, $\mathrm{PhD}^{2}$, Swapnil Pandey, $\mathrm{PhD}^{2}$, \\ Shivam Tiwari, $\mathrm{MSc}^{1}$, Lokendra Bahadur Yadav, $\mathrm{MSc}^{1}$, Akhilesh Kumar Shroti, MD, DM${ }^{1}$, \\ Manisha Vajpeyee, $\mathrm{PhD}^{1}$ \\ ${ }^{1}$ Department of Neurology, Pacific Medical College \& Hospital, Pacific Medical University, Udaipur, India \\ ${ }^{2}$ CSIR National Botanical Research Institute, Lucknow, India
}

Purpose: The purpose of this study was to assess the microbiota in middle cerebral artery thrombi retrieved in mechanical thrombectomy arising out of symptomatic carotid plaque within 6 hours of acute ischemic stroke. Thrombi were subjected to next-generation sequencing for a bacterial signature to determine their role in atherosclerosis.

Materials and Methods: We included 4 human middle cerebral artery thrombus samples (all patients were male). The median age for the patients was $51 \pm 13.6$ years. Patients enrolled in the study from Pacific Medical University and Hospital underwent mechanical thrombectomy in the stroke window period. All patients underwent brain magnetic resonance angiography (MRA) and circle of Willis and neck vessel MRA along with the standard stroke workup to establish stroke etiology. Only patients with symptomatic carotid stenosis and tandem lesions with ipsilateral middle cerebral artery occlusion were included in the study. Thrombus samples were collected, stored at -80 degrees, and subjected to metagenomics analysis.

Results: Of the 4 patients undergoing thrombectomy for diagnosis with ischemic stroke, all thrombi recovered for bacterial DNA in qPCR were positive. More than 27 bacteria were present in the 4 thrombus samples. The majority of bacteria were Lactobacillus, Stenotrophomonas, Pseudomonas, Staphylococcus, and Finegoldia.

Conclusion: Genesis of symptomatic atherosclerotic carotid plaque leading to thromboembolism could be either due to direct mechanisms like acidification and local inflammation of plaque milieu with lactobacillus, biofilm dispersion leading to inflammation like with pseudomonas fluorescence, or enterococci or indirect mechanisms like Toll 2 like signaling by gut microbiota.

Key Words: Metagenomics; Stroke; Microbiome; Thrombectomy; Thrombus

\section{INTRODUCTION}

Stroke is the second leading cause of death, and the leading cause of handicaps in the world. 'Atherosclerosis is one of the most critical pathways responsible for stroke. The production of atherosclerosis and stroke is associated with multiple internal and external (or environmental) influences. Since the microbiome in the human body outnumbers $\left(3.8 \times 10^{13}\right)$ human cells $\left(3 \times 10^{10}\right)$, there is a microbiome-dependent relationship to the host. This microbiota is like a second human genome influencing the onset, severity, and outcome of

\section{Correspondence to:} Atulabh Vajpeyee, MD, DM, FINS Department of Neurology, Pacific Medical College \& Hospital, Pacific Medical University, Bhilon ka Bedla Udaipur Rajasthan-313001, India Tel: +912943920000

Fax: +912943920081

E-mail: researchudr@gmail.com

Received: October 5, 2020 Revised: December 31, 2020 Accepted: January 4, 2021
Copyright $\odot 2021$ Korean Society of Interventional Neuroradiology

This is an Open Access article distributed under the terms of the Creative Commons Attribution Non-Commercial License (http://creativecommons.org/licenses/by-nc/4.0) which permits unrestricted non-commercial use, distribution, and reproduction in any medium, provided the original work is properly cited. 
a disease. Culture-independent techniques, like new generation sequencing (NGS), in the present era might help to explore bacterial signatures in a small sample compared to culture-dependent identification of bacteria.

Identified modifiable risk factors include smoking, obesity, hypercholesteremia, diabetes mellitus, and sedentary lifestyle, and numerous non-modifiable risk factors include age, sex, race, and genetics. Various infectious agents may cause direct inflammation. Second, it can lead to immune-mediated inflammation in the plaque, making it vulnerable to rupture. $^{3}$ Third, infection can occur at a distant site, which may induce a systematic inflammatory response. ${ }^{4,5}$ The fourth mechanism could be a low-grade virulent microorganism covered with biofilm, which could be dormant in the plaque of atherosclerosis, which may become active on specific triggers such as stress. ${ }^{6}$ Bacterial DNA in the intestinal oral cavity, skin, and plaque may be common, but may be associated with diabetes development, cardiovascular disease, and stroke. It is still uncertain if a particular bacterial profile is correlated with the extent of atherosclerosis in an atherosclerotic plaque or if it affects plaque stability. In samples with small quantities of bacterial DNA, such as atherosclerotic plaques, new culture-independent techniques have enabled the determination of a different class of bacteria. ${ }^{8}$

In this study, we used 165 sequencing (metagenomics analysis) of rRNA to characterize the bacterial DNA present in symptomatic patients' arteriosclerotic plaques. Multiple factors like blood flow hemodynamics decide plaque production at a given site, and different plaque characteristics predict vulnerability and risk of vascular events. Features associated with susceptibility to symptomatic events, such as stroke, include the presence of a thin or ulcerated fibrous cap, large lipid core, plaque inflammation, and intraplaque hemorrhage. ${ }^{9,10}$ The overall objective of this study was to determine the amount of bacterial DNA and microbial composition in the mechanically recovered middle cerebral artery thrombus samples in an acute stroke resulting from symptomatic carotid atherosclerotic plaque. In the last few years, the advent of mechanical thrombectomy along with the recovery of symptomatic thrombi without surgery has provided us with a unique opportunity to study microbial diversity.

\section{MATERIALS AND METHODS}

\section{Subjects}

As possible study subjects, patients with acute ischemic stroke $(n=4)$ with middle cerebral occlusion and symptomatic carotid stenosis, who were admitted to the Department of Neurology, Pacific Medical University \& Hospital between April 2018 and March 2019 were considered. The primary criterion for inclusion was diagnosis by neurologists of acute ischemic stroke with symptomatic carotid stenosis (more than $50 \%$ on carotid doppler) with occlusion of the middle cerebral artery. All presented within a 6 hour window to evaluate ischemic lesions, and brain magnetic resonance imaging (MRI) and/or computed tomography (CT) was done in all patients. They all had symptomatic carotid stenosis plaque with a thromboembolic event that led to artery occlusion at the middle cerebral artery. In addition to standard MRI of the brain, magnetic resonance angiography (MRA) of the neck and circle of Willis vessels was also performed. All of these carotid plaques were found to be vulnerable or unstable plaques on morphological evaluation by carotid Doppler and MRI plaque imaging. Exclusion criteria were as follows: admission $>6$ hours after the onset of the stroke, cardioembolic stroke, dissection-associated stroke, patent foramen ovale, vasculitis, history of malignancy, autoimmune disease, chronic hemodialysis kidney disease, Parkinson's disease; prior treatment of intravenous thrombolysis, cardioembolic stroke, hypercoagulant or genetic underlying cause of stroke, and fecal samples nonavailability. In total, the study cohort included 4 patients. The mean admission rate for the National Institutes of Health Stroke Scale (NIHSS) was 14.75 \pm 2.58 (range, 11 to 18). The Pacific Medical University \& Hospital Regional Review Board, Rajasthan, India has approved this study according to ethical standards. The regional review board has approved the use of human subjects for this study. All participants had given written, informed consent.

\section{Mechanical thrombectomy and thrombus sample collection}

All procedures were performed using biplane angiography (Allura xper FD 20-15; Phillips, India) cath lab. An introducer sheath was positioned on a femoral artery using the standard adapted seldinger technique. A guiding catheter with a tip balloon up to 9F was navigated into the carotid artery proximal to the occluded spot. The microcatheter with the guidewire (0.021 inches) was used to maneuver through the 
occluded site and over the thrombus to insert the stent retriever (Solitaire; Medtronic, Minneapolis, MN, USA). Solitaire revascularization devices were used to perform multiple retrieval runs, up to a maximum of 3 retrievals in the same vessel, and up to 2 retrievals per unit, respectively. The microcatheter was positioned across the visible clot, and the stent retrieval device was deployed by pulling the microcatheter back at the desired location. Where appropriate, Solitaire resheathing was done but not more than twice. Retrieval of thrombus was performed with negative aspiration with a $20 \mathrm{~mL}$ syringe. The final angiogram was done to assess flow restoration ( $\mathrm{TICl}$ flow). Repeated thrombectomy was done until a satisfactory angiographic result was achieved. For DNA extraction and qPCR analysis, the thrombi collected were divided into a $1.5 \mathrm{~mL}$ Eppendorf micro centrifugal tube.

\section{DNA extraction and quantification}

As directed by the manufacturer, the bacterial DNA was extracted from samples using a commercially available QIAamp DNA Mini Kit (Qiagen, Hilden, Germany). The quantity of DNA collected was measured using a Nanodrop spectrophotometer (Thermo Fisher Scientific Inc., Waltham, MA, USA).

\section{5 sequencing (metagenomics analysis)}

For the sequencing, 165 Amplicon-Seq hypervariable regions V1-V9 were amplified using primers, including the forward 165 primer 5'-ATCGCCTACCGTGAC-barcode-AGAGTTTGATCMTGGCTCAG-3' and Reverse 165 primer 5'-ATCGCCTACCGTGAC-barcode-CGGTTACCTTGTTACGACTT-3' with gene-specific sequences, MinION, Oxford Nanopore, and molecular barcodes. The PCR mixture for the full-length 165 rRNA gene (50 $\mu \mathrm{L}$ total volume) contained $10 \mathrm{ng}$ of DNA template $(10 \mu \mathrm{L}), 25 \mu \mathrm{L}$ long Amp Taq 2X master mix (NEB M0287), $1 \mu \mathrm{L} 16 \mathrm{~S}$ barcode (Barcode 01 to Barcode 04 each for a single sample separately), and $14 \mu \mathrm{L}$ nuclease-free water as indicated in the 165 barcoding kit (SQK-RAB204). The thermal PCR profile consisted of an initial denaturation of 60 seconds at $95^{\circ} \mathrm{C}$ followed by 25 cycles of 20 seconds at $95^{\circ} \mathrm{C}, 30$ seconds at $55^{\circ} \mathrm{C}, 2$ minutes at $65^{\circ} \mathrm{C}$, and a final stage of 5 minutes at $65^{\circ} \mathrm{C}$. The amplicons were washed with the Beckman Coulter (AMPure XP beads) using a ratio of $0.5 \mathrm{X}$. Usage of the Nanodrop spectrophotometer was evaluated for each sample quantity. The various bar-coded samples were pooled in equimolar ratio to get a final pool (100-150 ng in $10 \mu \mathrm{L}$ ) to do the sequencing library by adding $1 \mu \mathrm{L}$ of RAP (Rapid Annealing Primer) to the bar-coded DNA. The prepared sample
(11 $\mu \mathrm{L}$ DNA sample) was combined with Library Loading Beads $(25.5 \mu \mathrm{L})$ and Fuel Mix Running Buffer $(35.5 \mu \mathrm{L})$ and loaded onto SpotON Flow Cells Mk I (R9.4.1) (FLO-MIN106) for 12 hours using MinKNOWTM 19.06.8.

\section{Data availability}

Data that support the findings of this study are available upon reasonable request from the Pacific Medical University study investigators.

\section{RESULTS}

\section{Patient characteristics}

Within the study, all patients were male. The median age for the patients was $51 \pm 13.6$ years. All were subjected to mechanical thrombectomy during the window time of acute stroke with symptomatic carotid stenosis, with occlusion of the ipsilateral middle cerebral artery. All procedures were done within 6 hours of the onset of the stroke. All had recanalization of TICl 3 after the procedure. Two patients had a history of hypertension and 1 had diabetes mellitus. Before mechanical thrombectomy and thrombus sample collection, no patient had a history of serious infection, septicemia, or recent antibiotic treatment.

\section{Bacterial DNA found in aspirates of thrombus}

Of the 4 patients undergoing thrombectomy for diagnosis of ischemic stroke, all thrombi recovered for bacterial DNA in qPCR were positive. More than 27 bacteria were present in 4 thrombus samples. The majority of bacteria were Lactobacillus, Staphylococcus, and Stenotrophomonas in all 4 thrombi. Other abundant bacteria were Pseudomonas putida, Staphylococcus epidermidis, Staphylococcus hominis, and Finegoldia magna, as well as many others shown in Fig. 1. In fact, the majority of bacterial species in abundance in heat color map (Fig. 1) may reveal them to be commensal or low-grade pathogens around the human body. Their transgression into the bloodstream from the oral cavity, gut, or skin may render them to be lodged in carotid plaque during transient bacteremia without overt sepsis.

\section{DISCUSSION}

In the initial stage of a chronic inflammatory disease like 

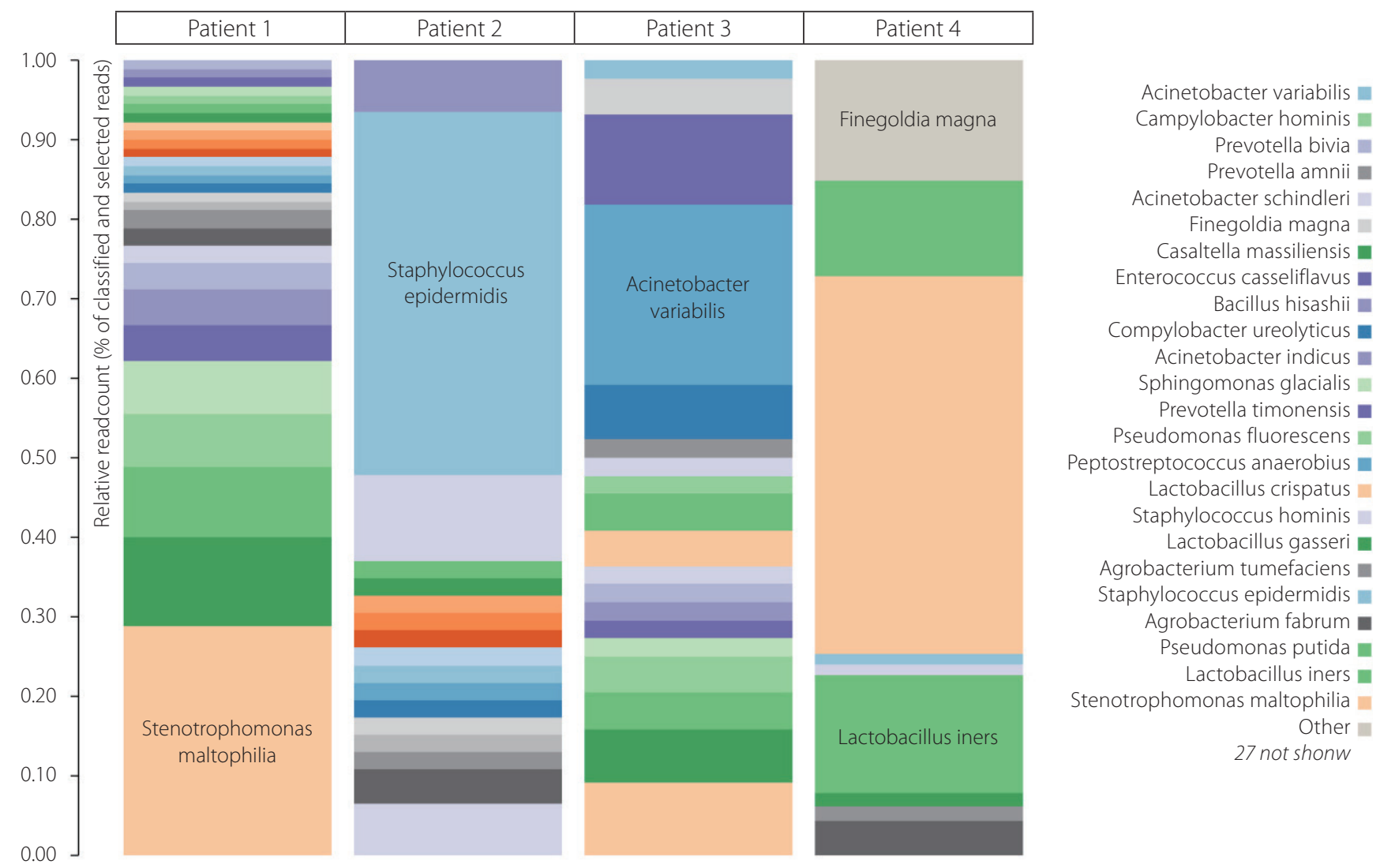

Fig. 1. Color heat maps of bacterial species with relative abundance. atherosclerosis, low-density lipoprotein (LDL) undergoes enzyme-modified oxidation into proinflammatory particles that induce an innate inflammatory intimal response.

At the extracellular matrix, familiar, smooth muscle cells also secrete proteoglycans, collagen, and elastic fibers. ${ }^{11}$ A thin-cap fibroatheroma develops and can rupture from some stimuli during the later stage of plaque formation. It is mainly by its composition that proteolytic enzyme activity occurs unchecked. This lesion is commonly called a fragile plaque because of the possibility of thromboembolic events and occlusion threatening rupture and life. ${ }^{12}$ These lesions peak at around the age of 55 to 65 years, coinciding with increased myocardial infarction and stroke incidence. ${ }^{13,14}$ In this study we found more than 27 bacteria through NGS in 4 thrombus samples, suggesting their presence in plaque may have been preceded in the past by single or repeated bacteremia. Since all of them are nonpathogenic or lowgrade pathogenic commensal in (oral cavity, gut, skin) and around the human body, these bacteria will not lead to frank sepsis or septicemia but only transient single or recurrent bacteremia that get lodged in various sites including carotid plaque. ${ }^{15}$ We intend to describe their plaque presence and their interplay with atherosclerotic plaque, which eventually turn a stable plaque into a vulnerable or symptomatic plaque that leads to thrombotic or thromboembolic events. The hallmarks of symptomatic ruptured plaque are a thin or ruptured fibrous cap, large lipid core, intraplaque hemorrhage, and active inflammation as evident on MRI plaque imaging with contrast. ${ }^{15}$ We know that most of these bacteria are commensal, non-pathogenic, or low pathogenic in and around the human body and transgress from their site to atherosclerotic plaque in some way without leading to overt infection in immunocompetent hosts. There could be various complex mechanisms of plaque-bacteria interaction. It consists of direct invasive bacterial activity, causing inflammation and rupture through proteolytic and other enzymes, acidification of the local intra-plaque environment leading to plaque lipid core growth, and inflammation through biofilm dispersal. ${ }^{16}$

Lactobacillus crispatus, Lactobacillus gasseri, Lactobacillus iners, and Lactobacillus jensenii are the different species known as Lactobacillus. It is a gram-positive, catalase-nega- 
tive, potentially anaerobic rod-shaped bacterium with strain type CCUG 28746T.17,18 As with other genera of the genus, the species Lactobacillus has the capacity to produce lactic acid and use it as a result of glucose metabolism. Due to the production of lactic acid, the presence of Lactobacillus in atherosclerotic plaque can produce an acidic milieu, and it is established that LDL oxidation by cells in the presence of iron is faster at acid $\mathrm{pH}$. Atherosclerotic lesions grow with the help of extensive $L D L$ oxidation with iron alone or cysteine much faster at acid $\mathrm{pH}$, even at a mild acid $\mathrm{pH}(\mathrm{pH} 6.5) .{ }^{19}$ Plasma contains iron that is produced using the transferrin protein. Lowering the $\mathrm{pH}$ associated with transferrin and transferrin also releases iron and catalyzes LDL oxidation. Ceruloplasmin is a major copper plasma carrier that becomes more effective in catalyzing the oxidation of LDLs at acid $\mathrm{pH}$, or even at $\mathrm{pH}$ 7.0. For this consequence, it is possible to see the concentrations of ceruloplasmin and transferrin below those found in plasma in atherosclerosis. This localized acid pH can help to understand why atherosclerotic lesions build up more in a few sites of the body, where LDL oxidation is severe. ${ }^{19}$

Lactobacillus has been known to prevent atherosclerosis largely by reducing trimethylamine oxide by modulation of gut dysbiosis. ${ }^{20}$ Few studies contradict this like Lactobacillus casei cell wall extract accelerated atherosclerosis in mice. ${ }^{21}$ Lactobacillus fermentum and L. ingluviei were associated with significant weight gain in humans. ${ }^{22}$ and we know obesity alters gut permeability to facilitate bacterial endotoxemia and promote chronic inflammation like atherosclerosis. In our study, we did not look for a metabolite and endotoxin assay.

Inflammation and infection can play a key role in the progression of atherosclerosis in the bacteria's ability to form biofilms within arterial plaques. ${ }^{6}$ Atherosclerosis progresses slowly, with LDL and plasma cholesterol accumulating under the endothelium of arterial walls resulting in atheromatous plaque. ${ }^{23}$ The key threat associated with atherosclerosis is the sudden rupture of a healthy atheroma, leading to life-threatening thrombotic and thromboembolic events. ${ }^{12}$ The value of plaque stabilization is highlighted by the finding that 76 percent of all fatal coronary thrombi were produced from arterial plaque rupture. ${ }^{24}$ We show that many bacterial species are identified in plaques that form biofilm and deposits in the carotid arterial plaque, stimulating the biofilm dispersion response in the presence of transferrin associated with physiologically relevant norepinephrine levels. The bacterial enzyme is capable of releasing dispersion of biofilm and forming surrounding biofilm micro-colonies and allowing bacteria to escape the biofilm matrix. Biofilm dispersion is characterized by bacteria trapping and removing degrading enzymes from the biofilm matrix to release individual cells and a transition to higher growth rates. ${ }^{25,26}$ Interestingly, when suddenly limiting the amount of nutrients increases, bacteria biofilm can be used to respond to the event of mounting dispersion. ${ }^{25}$ Biofilm forming bacteria such as Pseudomonas fluorescens, enterococcus are known to elevate factor level VIII, are nonpathogenic motile, or are low-grade pathogens that usually increase the risk of stroke. ${ }^{27}$ An essential nutrient released from its bound state like free iron production, using high norepinephrine promotes biofilm dispersion events especially in response to stress. ${ }^{25}$

Sternotrophomonas Maltophilia is low-virulence and generates biofilm. ${ }^{28}$ The S. maltophilia protease coded for by the StmPr1 gene is capable of breaking down collagen, fibronectin, and fibrinogen protein components and may thus lead to local tissue damage and hemorrhage. ${ }^{29}$ F. Nucleatum in periodontal illness is associated with the development of Lemierre's syndrome, which is an infection of the upper respiratory tract along with septic thrombophlebitis of the inner and outer jugular veins, arteritis, and aneurysms. ${ }^{30-32}$

Staphylococcus hominis is coagulase-negative staphylococci that primarily colonize axillary and pubic regions that abound in apocrine glands. Low-grade Staphylococcal hominis bacteremia may remain asymptomatic, but may result in deposition of the organism into atheromatous plaques that trigger local inflammation and transform it into unstable or ruptured plaque. ${ }^{33}$

Brevundimonas vesicularis, found in and around the gut and genitourinary tract and bloodstream infections, is implicated in the production of multiple infections. ${ }^{34}$ Mice studies provide another possible mechanism relating to the development of atherosclerosis and vascular events in the microorganism. The hepatic endothelium of the mice microorganism induces synthesis of Von Willebrand factor (VWF), which has been shown to promote thrombus production in the ligation-injured carotid artery. Microbial products that translocate from the gut lumen to the liver, where they stimulate Toll-like receptors, resulting in increased VWF synthesis and release into the circulation. The elevated level of VWF in plasma stimulates platelet deposition and thrombus production in the carotid artery. ${ }^{35}$

In addition to standard risk factors such as hypertension, smoking, diabetes, hypercholesterolemia, obesity, and 
lifestyle, microbiota in and around the human body can be a risk factor for atherosclerotic diseases such as stroke, coronary artery disease, and peripheral vascular diseases. In the current COVID-19 pandemic, it reinforces the one-word Vidhra, an old Sanskrit word with a varied meaning such as cleanliness, free of spots or stains, sunlight, fire, pure wind, where all meanings pointing to good hygiene to prevent atherosclerotic diseases as well. Possible use of antibiotics or probiotics requires larger trials to provide any definite outcome. The limitations of this study are its small sample size, lack of control data, and the contradictory data about lactobacillus in previous reports.

\section{CONCLUSION}

The genesis of symptomatic atherosclerotic carotid plaque leading to thromboembolism could be either due to direct mechanisms like acidification and local inflammation of plaque milieu with lactobacillus, biofilm dispersion leading to inflammation like with pseudomonas fluorescence, Enterococci or indirect mechanisms like Toll 2 like signaling by gut microbiota. This necessitates a larger study that correlates thrombus microbiota and gut microbiota to verify surrounding microbiota as additional risk factors for stroke.

\section{Fund}

The authors acknowledge the Pacific Medical University and Hospital, for providing facilities and support during the study. The authors acknowledge the financial assistance from the Department of Science and Technology, Government of Rajasthan, India.

\section{Ethics Statement}

The Pacific Medical University \& Hospital Regional Review Board, Rajasthan, India has approved this study according to ethical standards. The regional review board has approved the use of human subjects for this study.

\section{Conflicts of Interest}

The authors have no conflicts to disclose.

\section{Author Contributions}

Concept and design: AV and MV. Analysis and interpretation: PSC, SP, ST, LBY, AKS, and MV. Data collection: SP, ST, and LBY. Writing the article: AV, PSC, SP, AKS, and MV. Critical revision of the article: AKS. Final approval of the article: MV. Statistical analysis: AKS. Obtained funding: AV.

\section{ORCID}

Atulabh Vajpeyee: https://orcid.org/0000-0002-7942-4302

Puneet Singh Chauhan: https://orcid.org/0000-0001-5955-8656

Swapnil Pandey: https://orcid.org/0000-0001-5086-709X

Shivam Tiwari: https://orcid.org/0000-0001-9465-1215

Lokendra Bahadur Yadav: https://orcid.org/0000-0002-5035-0573

Akhilesh Kumar Shroti: https://orcid.org/0000-0003-0422-520X

Manisha Vajpeyee: https://orcid.org/0000-0001-5010-2863

\section{REFERENCES}

1. Johnson W, Onuma O, Owolabi M, Sachdev S. Stroke: a global response is needed. Bull World Health Organ 2016;94:634-634A

2. Sender R, Fuchs S, Milo R. Revised estimates for the number of human and bacteria cells in the body. PLoS Biol 2016;14:e1002533

3. Epstein SE, Zhu J, Burnett MS, Zhou YF, Vercellotti G, Hajjar D. Infection and atherosclerosis: potential roles of pathogen burden and molecular mimicry. Arterioscler Thromb Vasc Biol 2000;20:1417-1420

4. Libby P, Egan D, Skarlatos S. Roles of infectious agents in atherosclerosis and restenosis: an assessment of the evidence and need for future research. Circulation 1997;96:4095-4103

5. Rosenfeld ME, Campbell LA. Pathogens and atherosclerosis: update on the potential contribution of multiple infectious organisms to the pathogenesis of atherosclerosis. Thromb Haemost 2011;106:858-867

6. Lanter BB, Sauer K, Davies DG. Bacteria present in carotid arterial plaques are found as biofilm deposits which may contribute to enhanced risk of plaque rupture. mBio 2014;5:e01206-14

7. Amar J, Serino M, Lange C, Chabo C, lacovoni J, Mondot S, et al.; D.E.S.I.R. Study Group. Involvement of tissue bacteria in the onset of diabetes in humans: evidence for a concept. Diabetologia 2011;54:3055-3061

8. Koren O, Spor A, Felin J, Fåk F, Stombaugh J, Tremaroli V, et al. Human oral, gut, and plaque microbiota in patients with atherosclerosis. Proc Natl Acad Sci U S A 2011;108 (Supplement 1):4592-4598

9. Yilmaz A, Lipfert B, Cicha I, Schubert K, Klein M, Raithel D, et al. Accumulation of immune cells and high expression of chemokines/chemokine receptors in the upstream shoulder of atherosclerotic carotid plaques. Exp Mol Pathol 2007;82:245-255 
10. Fagerberg B, Ryndel M, Kjelldahl J, Akyürek LM, Rosengren $L$, Karlström L, et al. Differences in lesion severity and cellular composition between in vivo assessed upstream and downstream sides of human symptomatic carotid atherosclerotic plaques. $J$ Vasc Res 2010;47:221-230

11. Kany S, Vollrath JT, Relja B. Cytokines in inflammatory disease. Int J Mol Sci 2019;20:6008

12. Falk E, Shah PK, Fuster V. Coronary plaque disruption. Circulation 1995:92:657-671

13. Loria V, Cosentino N, Montone RA, Niccoli G. Biomarkers and coronary atherosclerotic burden and activity as assessed by coronary angiography and intra-coronary imaging modalities. In: Branislav B. Coronary angiography. London: IntechOpen, 2011

14. Aziz M, Yadav KS. Pathogenesis of atherosclerosis a review. Med Clin Rev 2016;2:22

15. Mughal MM, Khan MK, DeMarco JK, Majid A, Shamoun F, Abela GS. Symptomatic and asymptomatic carotid artery plaque. EXpert Rev Cardiovasc Ther 2011;9:1315-1330

16. Sauer K, Cullen MC, Rickard AH, Zeef LA, Davies DG, Gilbert P. Characterization of nutrient-induced dispersion in Pseudomonas aeruginosa PAO1 biofilm. J Bacteriol 2004;186:7312-7326

17. Mitsuoka T. The human gastrointestinal tract. In: Wood BJB. Lactic acid bacteria in health and disease. Boston: Springer, 1992:69-114

18. Husni RN, Gordon SM, Washington JA, Longworth DL. Lactobacillus bacteremia and endocarditis: review of 45 cases. Clin Infect Dis 1997;25:1048-1055

19. Leake DS. Does an acidic pH explain why low density lipoprotein is oxidised in atherosclerotic lesions? Atherosclerosis 1997;129:149-157

20. Janeiro MH, Ramírez MJ, Milagro Fl, Martínez JA, Solas M. Implication of trimethylamine $\mathrm{N}$-oxide (TMAO) in disease: potential biomarker or new therapeutic target. Nutrients 2018;10:1398

21. Chen S, Lee Y, Crother TR, Fishbein M, Zhang W, Yilmaz A, et al. Marked acceleration of atherosclerosis after Lactobacillus casei-induced coronary arteritis in a mouse model of Kawasaki disease. Arterioscler Thromb Vasc Biol 2012;32:e60-e71

22. Million M, Angelakis E, Paul M, Armougom F, Leibovici L, Raoult D. Comparative meta-analysis of the effect of Lactobacillus species on weight gain in humans and animals. Microb Pathog 2012:53:100-108

23. Lusis AJ. Atherosclerosis. Nature 2000;407:233-241
24. Falk E. Pathogenesis of atherosclerosis. J Am Coll Cardiol 2006:47(8 Suppl):C7-C12

25. McDougald D, Rice SA, Barraud N, Steinberg PD, Kjelleberg S. Should we stay or should we go: mechanisms and ecological consequences for biofilm dispersal. Nat Rev Microbiol 2011;10:39-50

26. Boyd A, Chakrabarty AM. Role of alginate lyase in cell detachment of Pseudomonas aeruginosa. Appl Environ Microbiol 1994;60:2355-2359

27. Shaban A, Hymel B, Chavez-Keatts M, Karlitz JJ, Martin-Schild S. Recurrent posterior strokes in inflammatory bowel disease patients. Gastroenterol Res Pract 2015;2015:672460

28. Flores-Treviño S, Bocanegra-lbarias P, Camacho-Ortiz A, Morfín-Otero R, Salazar-Sesatty HA, Garza-González E. Stenotrophomonas maltophilia biofilm: its role in infectious diseases. Expert Rev Anti Infect Ther 2019;17:877-893

29. Looney WJ, Narita M, Mühlemann K. Stenotrophomonas maltophilia: an emerging opportunist human pathogen. Lancet Infect Dis 2009;9:312-323

30. Ford PJ, Gemmell E, Chan A, Carter CL, Walker PJ, Bird PS, et al. Inflammation, heat shock proteins and periodontal pathogens in atherosclerosis: an immunohistologic study. Oral Microbiol Immunol 2006;21:206-211

31. Pyysalo MJ, Pyysalo LM, Pessi T, Karhunen PJ, Öhman JE. The connection between ruptured cerebral aneurysms and odontogenic bacteria. J Neurol Neurosurg Psychiatry 2013;84:12141218

32. Elkaïm R, Dahan M, Kocgozlu L, Werner S, Kanter D, Kretz JG, et al. Prevalence of periodontal pathogens in subgingival lesions, atherosclerotic plaques and healthy blood vessels: a preliminary study. J Periodontal Res 2008;43:224-231

33. Renko J, Lepp PW, Oksala N, Nikkari S, Nikkari ST. Bacterial signatures in atherosclerotic lesions represent human commensals and pathogens. Atherosclerosis 2008;201:192-197

34. Gilad J, Borer A, Peled N, Riesenberg K, Tager S, Appelbaum A, et al. Hospital-acquired brevundimonas vesicularis septicaemia following open-heart surgery: case report and literature review. Scand J Infect Dis 2000;32:90-91

35. Jäckel S, Kiouptsi K, Lillich M, Hendrikx T, Khandagale A, Kollar B, et al. Gut microbiota regulate hepatic von Willebrand factor synthesis and arterial thrombus formation via Toll-like receptor-2. Blood 2017;130:542-553 